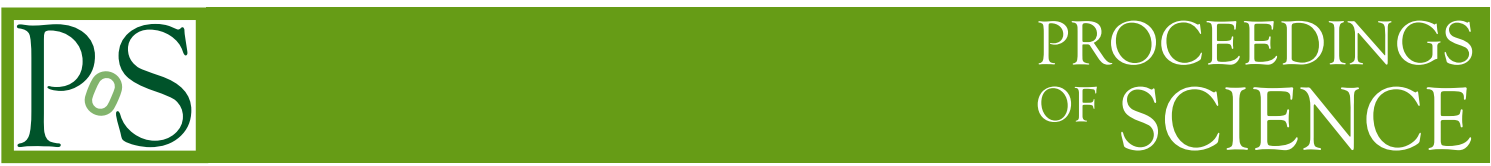

\title{
The Phase-2 upgrade of the CMS Outer Tracker
}

\author{
Martin Lipinski ${ }^{a, 1, *}$ \\ ${ }^{a}$ I. Physikalisches Institut B, RWTH Aachen University, \\ Sommerfeldstr. 14, 52074 Aachen, Germany \\ E-mail: mlipinski@physik.rwth-aachen.de
}

The Large Hadron Collider at CERN will undergo a major upgrade in the Long Shutdown 3 from 2025-2027. The so-called High Luminosity LHC (HL-LHC) is expected to deliver peak instantaneous luminosities of about $5-7.5 \times 10^{34} \mathrm{~cm}^{-2} \mathrm{~s}^{-1}$ and an integrated luminosity of about $3000-4500 \mathrm{fb}^{-1}$ during ten years of operation. In order to fully exploit the delivered luminosity and to cope with the demanding operating conditions, the entire silicon tracking system of the CMS experiment will have to be replaced. The Phase-2 Outer Tracker (OT) will have an increased radiation hardness, a higher granularity, and will be able to cope with larger data rates. In addition, the OT will provide tracking information to the Level-1 trigger. To achieve this, each module will consist of two closely spaced sensors. The readout chips use hit information of both sensors for a rough transverse momentum measurement. This novel concept will improve the selection performance of the trigger under large pileup conditions. Furthermore, the Phase-2 OT will use evaporative $\mathrm{CO}_{2}$ cooling and a DC-DC conversion based powering scheme to keep the material budget small. In this report, the design of the CMS Phase-2 Outer Tracker and the present status of the project will be presented.

\footnotetext{
*** The European Physical Society Conference on High Energy Physics (EPS-HEP2021), ***

*** 26-30 July $2021 * * *$

*** Online conference, jointly organized by Universität Hamburg and the research center DESY ***
}

${ }^{1}$ For the CMS collaboration.

* Speaker 


\section{Introduction}

The CMS detector [1] at the CERN LHC features a silicon tracker as its innermost device. It comprises a pixel detector and a strip tracker that provide space point measurements of charged particles' trajectories. The original tracker was developed for a maximum instantaneous luminosity of $1 \times 10^{34} \mathrm{~cm}^{-2} \mathrm{~s}^{-1}$, corresponding to 25 simultaneous proton-proton interactions per bunch crossing (pileup) on average. In 2017, the CMS Collaboration has replaced the pixel detector with an upgraded one [2], which is able to cope with the luminosity of about $2 \times 10^{34} \mathrm{~cm}^{-2} \mathrm{~s}^{-1}$ reached by the LHC at that time. In light of the High-Luminosity LHC (HL-LHC) upgrade, the CMS collaboration plans to replace the entire tracking system during the Long Shutdown 3 in 2025 2027 as part of the Phase-2 upgrade [3]. The HL-LHC will deliver instantaneous luminosities of $5-7.5 \times 10^{34} \mathrm{~cm}^{-2} \mathrm{~s}^{-1}$, resulting in an average pileup of $140-200$. A total amount of about $3000-4000 \mathrm{fb}^{-1}$ will be delivered.

Main goals of the tracker upgrade are a higher granularity and an increased readout bandwidth to be able to deal with larger hit and trigger rates. Furthermore, an increased radiation tolerance is required to sustain a good tracking performance with fluences of up to $2.3 \times 10^{16} \mathrm{n}_{\mathrm{eq}} / \mathrm{cm}^{2}$ and a total ionizing dose of up to $1.2 \mathrm{Grad}$. The tracking acceptance in the forward region will be extended from a pseudorapidity of $|\eta|<2.5$ to $|\eta|<4$. The Phase-2 tracker is composed of two parts, a silicon pixel detector (Inner Tracker) and a silicon strip and macro-pixel detector (Outer Tracker). This report focuses on the design of the Outer Tracker. More information about the Inner Tracker can be found in [2].

A main design driver of the Outer Tracker is its requirement to contribute tracking information to the Level-1 trigger, which presently only uses information from the calorimeters and the muon system. The use of tracking information will improve the selection performance of the trigger under large pileup conditions. However, a readout of the whole detector at the bunch crossing frequency of $40 \mathrm{MHz}$ is not possible due to bandwidth limitations. Therefore, a novel module concept has been developed, that allows a data reduction at the front-end by discriminating tracks based on their transverse momentum $\left(p_{\mathrm{T}}\right)$. The concept is shown in Figure 1. Each module consists of two closely spaced silicon sensors that are connected to the same readout chips. Once a particle traverses the module, the readout electronics compares the hit position in both silicon sensors. If the hit in the
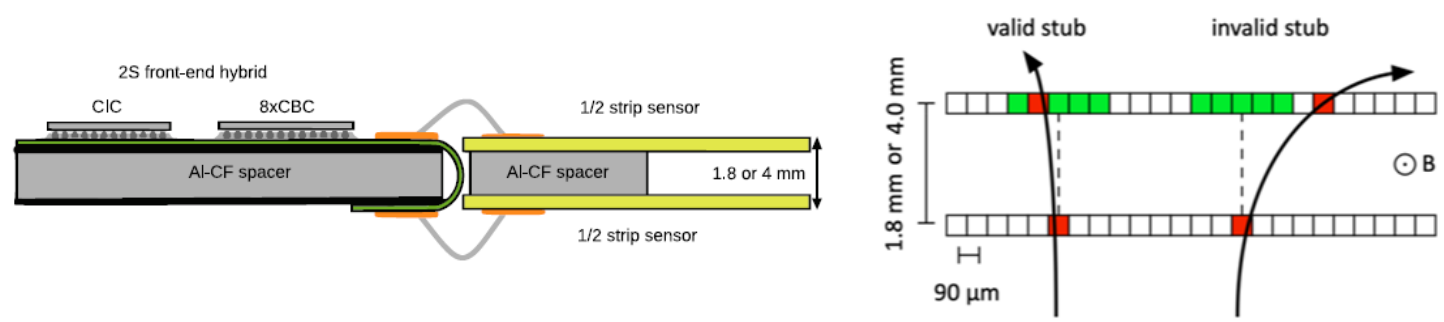

Figure 1: Illustration of the module concept. Left: a cross section of one half of the module, showing the two silicon sensors (yellow), which are connected to the flex PCB of the front-end hybrid (green) with wire bonds (gray) [3]. Right: schematic, enlarged view of the two silicon sensors with indicated trajectories of two particles. Strips with a hit are red; the search windows in the top sensor are indicated in green. The $p_{\mathrm{T}}$ of the right track is too low to create a valid stub. 


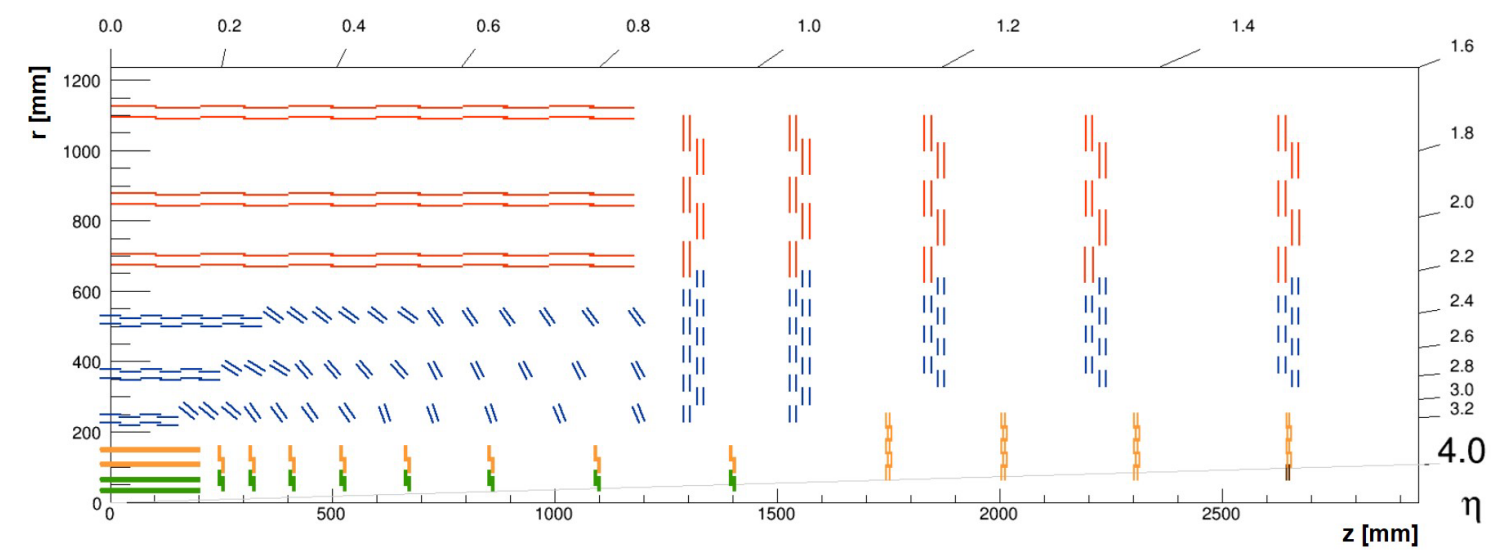

Figure 2: Layout of the Phase-2 tracker [3]. Colored lines represent the detector modules. The Outer Tracker modules are shown in red (2S) and blue (PS), whereas pixel modules of the Inner Tracker are shown in yellow and green. One quarter of the detector is shown.

second sensor is located within a search window relative to the hit in the first sensor, a tracklet information (called stub) is generated and sent to the trigger. The idea behind this concept is that the hit position in the second sensor depends on the curvature of the track in the $3.8 \mathrm{~T}$ magnetic field of CMS and therefore on the transverse momentum of the particle. Only tracks above a certain $p_{\mathrm{T}}$-threshold are selected, resulting in a significant data reduction. The threshold can be adjusted by changing the programmable width of the search window, and varies between $2-3 \mathrm{GeV}$. As the data reduction is performed directly on the modules, the stub information can be sent to the trigger at the bunch crossing frequency of $40 \mathrm{MHz}$. Only in case the Level-1 trigger selects an event, the entire detector is read out. The typical Level-1 trigger frequency will be around $750 \mathrm{kHz}$.

\section{Layout of the Phase-2 Outer Tracker}

A layout of the tracker is shown in Figure 2. The central part of the Outer Tracker consists of a cylindrical barrel with six layers of detector modules. The innermost three layers have a section with modules that are progressively tilted along the forward direction. The detector is complemented by one endcap per side with five layers of modules that cover pseudorapidities up to $|\eta| \approx 2.8$. In total there will be 7608 modules with two strip sensors ( $2 \mathrm{~S}$ modules) and 5592 modules with a strip and a macro-pixel sensor (PS modules). The $2 \mathrm{~S}$ modules will be used at radii larger than $60 \mathrm{~cm}$, whereas the PS modules will be used for smaller radii. The total silicon area of the Outer Tracker is about $190 \mathrm{~m}^{2}$ with 213 million channels.

\section{Module Design}

The $2 \mathrm{~S}$ modules consist of two $10 \times 10 \mathrm{~cm}^{2}$ large $\mathrm{n}$-in-p strip sensors with a strip pitch of $90 \mu \mathrm{m}$. Each sensor is divided into two halves, corresponding to a strip length of $5 \mathrm{~cm}$. The spacing of the two silicon sensors is $1.8 \mathrm{~mm}$ or $4.0 \mathrm{~mm}$ depending on the module position in the detector. For the PS modules n-in-p sensors are used as well. The strip sensor has a size of $5 \times 10 \mathrm{~cm}^{2}$. It is also divided into two parts, with a strip length of $2.4 \mathrm{~cm}$ and a pitch of $100 \mu \mathrm{m}$. The PS pixel sensor 

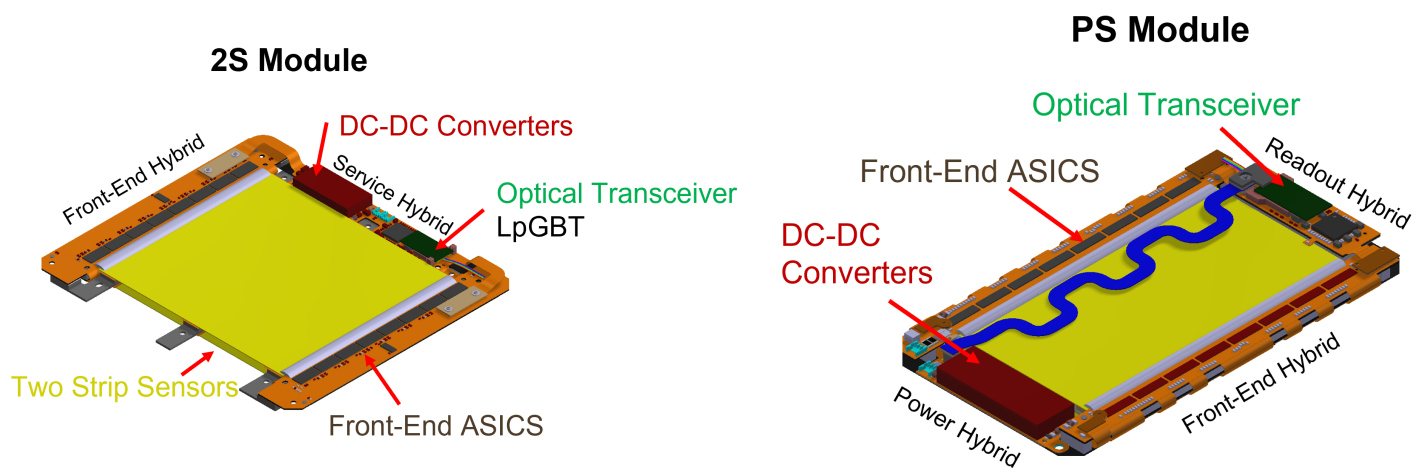

Figure 3: Schematic views of the $2 \mathrm{~S}$ module (left) and the PS module (right). The DC-DC converters are below the electromagnetic shield, which is indicated in red.

features about 30000 macro pixels with a size of $1.5 \mathrm{~mm} \times 100 \mu \mathrm{m}$. Sensor spacings of $1.6 \mathrm{~mm}$, $2.6 \mathrm{~mm}$, and $4.0 \mathrm{~mm}$ have been chosen for the PS modules.

Figure 3 shows schematic views of both module types. The sensors are glued to spacers made from carbon fiber reinforced aluminum. The strip sensors are wire-bonded to the Front-End Hybrids (FEHs) and are read out from both sides [3]. In case of the $2 \mathrm{~S}$ module the readout is done by the CMS Binary Chip (CBC) [? ]. The CBC has 254 channels, of which 127 are connected to the top sensor and 127 are connected to the bottom sensor. The correlation logic that builds the stubs is integrated into the chip. The readout is binary with a programmable signal threshold and runs at $320 \mathrm{MHz}$. Eight CBCs are required to read out one half module. In contrast, the PS module requires two different readout chips, as the bottom sensor is pixelated. The strip sensor is read out by eight Short Strip ASICs (SSA) per side, whereas 16 Macro Pixel ASICs (MPA) are bump-bonded to the pixel sensor. The correlation logic is integrated into the MPA, which receives the data from the SSAs through the folded front-end hybrid. The front-end hybrids of both module types each use one Concentrator Integrated Circuit (CIC) to collect the data from the readout chips. The CICs perform a sparsification (zero suppression) and send the data to the low-power Gigabit Transceiver (LpGBT) [3] on the service hybrid (2S modules) or on the readout hybrid (PS modules). The LpGBT sends the data to and receives clock, trigger and programming signals from the detector back-end. The Versatile Transceiver+ (VTRx+) is used for the opto-electrical conversion on the module. DC-DC converters for the powering of the module, the VTRx+, and the LpGBT are integrated into the service hybrid of the $2 \mathrm{~S}$ module, whereas for the PS modules this functionality is split into the power hybrid and the readout hybrid. Each module of the Phase-2 Outer tracker is a self-contained unit with a direct connection to the detector back-end (low and high voltage, optical fiber). There are no other boards or electronics needed in the active volume.

\section{Powering}

As a result of the increased granularity and the more complex readout electronics, the power consumption of the Phase-2 Outer Tracker will increase significantly compared to the original detector. An increase from $33 \mathrm{~kW}$ to $85 \mathrm{~kW}$ total front-end power is predicted. In order to keep 
power losses and voltage drops on the $40 \mathrm{~m}$ long supply cables at an acceptable level, a powering scheme using DC-DC converters has been chosen.

The $2 \mathrm{~S}$ modules implement a two-stage DC-DC conversion. The first stage uses the bPOL12V DC-DC buck converter ASIC. It converts the module input voltage of $\approx 10 \mathrm{~V}$ down to $2.55 \mathrm{~V}$, which is required by the VTRX+. This voltage is also used as the input for the second stage, consisting of a bPOL2V5 DC-DC converter. The second stage provides an output voltage of $1.25 \mathrm{~V}$ for the CBCs, CICs, the LpGBT, and the VTRx+, which requires two input voltages. The powering of the PS module is similar; however, the second stage consists of two bPOL2V5 DC-DC converters that provide $1.25 \mathrm{~V}$ and $1.0 \mathrm{~V}$, which are required by the MPA, SSA, and CIC chips. On both modules a shield made of a $150 \mu \mathrm{m}$ thick aluminum foil is used to reduce the electromagnetic emissions of the DC-DC converters. The bPOL12V and bPOL2V5 are radiation tolerant DC-DC buck converters that were developed by the CERN Electronic Systems for Experiments group [4].

Using a DC-DC conversion powering scheme lowers the supply currents on the cables by the conversion ratio $r$, resulting in a reduction of the voltage drop by $r$ and of the power loss by $r^{2}$. Assuming a typical conversion efficiency of about $80 \%$ per DC-DC stage, and taking voltage drops along the supply lines into account, the module supply currents are reduced by a factor of approximately 5 compared to a conventional powering scheme.

\section{Mechanics and Cooling}

Modules are held in place by lightweight support structures made from carbon fiber and foam. In the endcaps they are mounted on half-disks, with four half-disks forming one detector layer. In the barrel they are mounted on so-called ladders (2S) and planks (PS) oriented along the beam axis. The PS modules of the tilted barrel section (left side of Figure 4) are mounted on rings. The tilted section not only reduces the material budget because particles at high pseudorapidities travel a shorter path through the silicon, but it also increases the efficiency of the stub-finding, as indicated in Figure 4 on the right. Stubs can only be built if the hits in both sensors of a module are in the same sensor half. A correlation between the left and right FEH of a module or even between two modules is not possible. For high- $\eta$ tracks the offset between hits in both sensors is smaller in a tilted geometry compared to a flat geometry, therefore the probability of having hits in two different sensor halves is also smaller.

The Phase- 2 tracker will be operated at a temperature of about $-35^{\circ} \mathrm{C}$ using a bi-phase $\mathrm{CO}_{2}$ cooling system. The $2 \mathrm{~S}$ modules will be screwed to cooling inserts that are in contact with thin cooling pipes of $2 \mathrm{~mm}$ inner diameter and $150 \mu \mathrm{m}$ wall thickness. Recent thermal simulations indicate that six cooling contacts per module are required for a proper cooling. The PS modules will be glued to the support structure for a better thermal contact.

\section{Prototyping and Testing}

The project is currently in the late prototype phase. The status of the $2 \mathrm{~S}$ modules is well advanced, with the final version of the CIC being about to be submitted, the final version of the $\mathrm{CBC}$ being in mass production, and the pre-production of the silicon sensors having started. The latest versions of the front-end chips of the PS modules have been or are about to be submitted. 

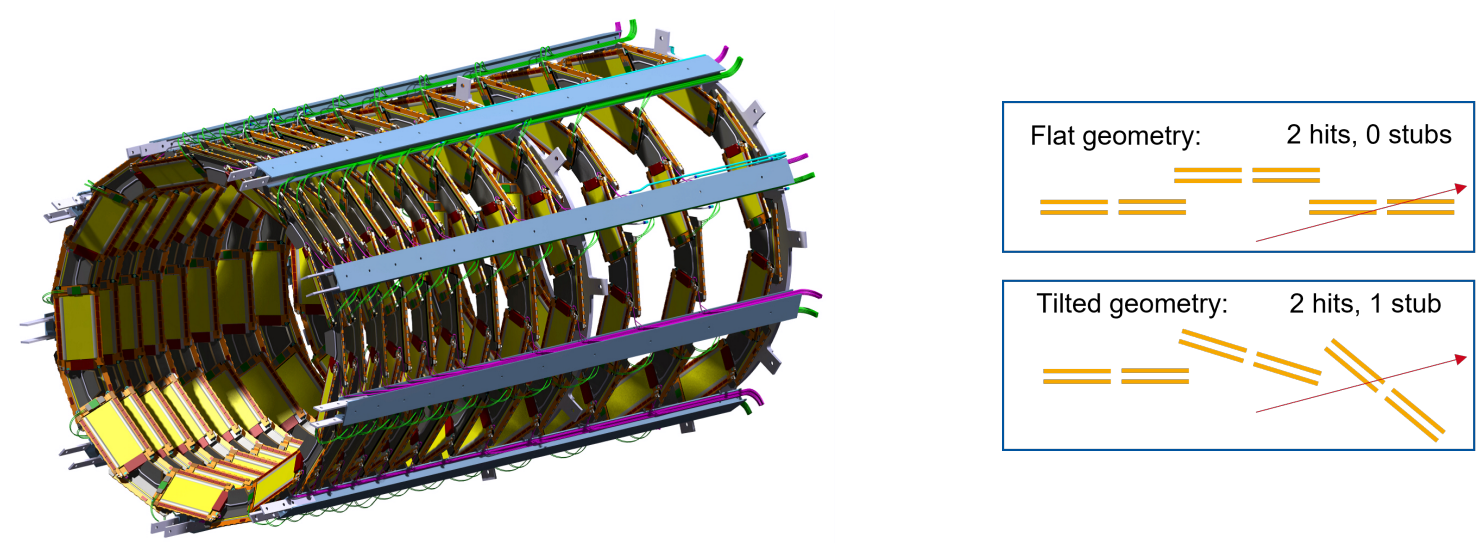

Figure 4: Left: CAD drawing of a tilted barrel section of the Outer Tracker [3]. Right: comparison of a flat and a tilted geometry. The red arrow indicates a track with a high pseudorapidity. In a flat geometry this track may hit a top sensor and a bottom sensor that are read out by different front-end hybrids, thus a stub information cannot be generated. In a tilted geometry both hits are detected by the same hybrid, and the stub information is generated.

About 30 prototypes of $2 \mathrm{~S}$ modules have been built, with the most recent ones using final prototypes of the service hybrids and the front-end hybrids, as shown in Figure 5. It was demonstrated that the requirements on the assembly accuracy can be met. Furthermore, the full readout chain has been exercised in several system tests and beam tests. For example, the stub finding mechanism has been proven to work [5]. It was also demonstrated that the electrical noise of the $2 \mathrm{~S}$ modules is below 1000 electrons, as specified, and that the two stage DC-DC conversion powering does not increase the module noise compared to a conventional powering. Furthermore, high rate tests with X-rays and injected pulses have shown a detection efficiency of $100 \%$ at a hit rate of $8 \mathrm{MHz} / \mathrm{cm}^{2}$ [6], which is the highest expected hit rate for $2 \mathrm{~S}$ modules. And finally, the module performed as expected during a test carried out in a $3 \mathrm{~T}$ magnetic field at the CERN M1 magnet. First PS modules have been built and are under evaluation. The series production of modules is expected to start in 2022 and to take about two years.

The sensors, spacers, and hybrids are glued into the module, making repairs almost impossible. Therefore, $100 \%$ of these components will be extensively tested during series production. The silicon sensors will be tested by the manufacturer, and are then sent to the sensor reception centers where they will undergo an extensive inspection. All hybrids will be tested at the respective manufacturer, including a passive thermal cycling and a quick electrical test. Reception tests will be performed at CERN and the institutes that build modules, including visual inspection and electrical tests. A high throughput test system was developed to qualify the approximately 45000 hybrids needed for the detector. Main components of this system are a backplane that is installed in a $3 \mathrm{U}$ Eurocrate, as well as several test cards, one for each hybrid type. Up to 12 test cards holding one hybrid each are plugged into one crate. The test system will be installed in a climatic chamber, which allows testing at the planned operating temperature of $-35^{\circ} \mathrm{C}$. Prototypes of the backplane and different test cards are available, and their functionality has been verified. The mass production of the test systems will start in 2021.

Every module will undergo a final test before it is assembled onto the detector support structures. 

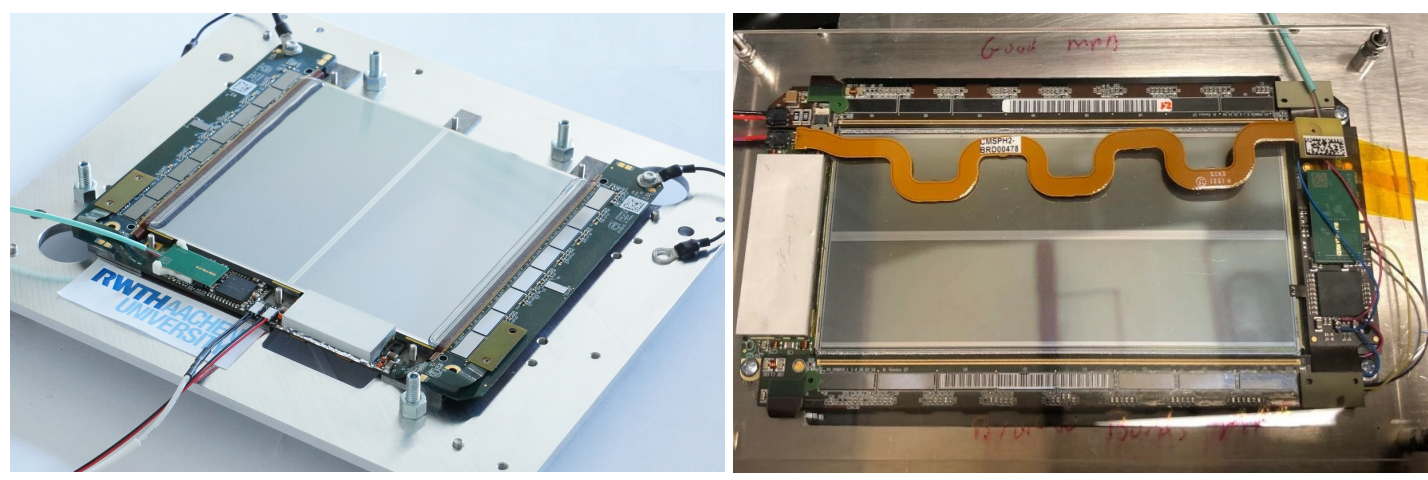

Figure 5: Photographs of a $2 \mathrm{~S}$ module prototype (left) and a PS module prototype (right).

A burn-in system, consisting of a cold box that is cooled by a liquid chiller, will be used for thermal cycling and for testing the functionality of up to 10 modules in parallel.

\section{Conclusions}

The Phase-2 upgrade of the CMS silicon tracker is an important project to ensure a good performance of the CMS detector after the High Luminosity LHC upgrade. The requirement to contribute tracking information to the Level-1 trigger is the main design driver of the Outer Tracker. An increased granularity, faster readout electronics, and a more radiation tolerant design will ensure a performance at least as good as the current tracker, even under much harsher conditions. The design utilizes state of the art technologies such as point of load DC-DC converters and an evaporative $\mathrm{CO}_{2}$ cooling system. The pre-production phase of several components has started. Test systems and procedures are currently being finalized in preparation for the series production.

\section{References}

[1] CMS Collaboration, The CMS experiment at the CERN LHC, 2008 JINST 3 S08004.

[2] Tracker Group of the CMS Collaboration, The CMS Phase-1 pixel detector upgrade, 2021 JINST 16 P02027.

[3] CMS Collaboration, The Phase-2 Upgrade of the CMS Tracker, CERN-LHCC-2017-009 (2017).

[4] CERN, DC-DC converter project website, http://cern.ch/project-DCDC-new.

[5] Tracker Group of the CMS collaboration, Beam test performance of prototype silicon detectors for the Outer Tracker for the Phase-2 Upgrade of CMS, 2020 JINST 15 P03014.

[6] O. Gries, Hochratentests mit Röntgenstrahlung an einem Prototypen des 2S-Moduls für das CMS Phase-2 Upgrade, Bachelor Thesis, RWTH Aachen University, 2021. 